\title{
RECENT WORKS ON MICROBES AND INFECTIONS IN CHINA
}

\author{
Selected from the Journal of \\ Microbes and Infections (China)
}


This page intentionally left blank 


\title{
RECENT WORKS ON MICROBES AND INFECTIONS IN CHINA
}

\author{
Selected from the Journal of \\ Microbes and Infections (China)
}

edited by

Yu-Mei Wen

Fudan University, China

Shan Lu

University of Massachusetts Worcester, USA

Yi-Wei Tang

Vanderbilt University, USA

Xin-Hua Weng

Fudan University, China 


\section{Published by}

World Scientific Publishing Co. Pte. Ltd.

5 Toh Tuck Link, Singapore 596224

USA office: 27 Warren Street, Suite 401-402, Hackensack, NJ 07601

UK office: 57 Shelton Street, Covent Garden, London WC2H 9HE

\section{British Library Cataloguing-in-Publication Data}

A catalogue record for this book is available from the British Library.

\section{RECENT WORKS ON MICROBES AND INFECTIONS IN CHINA Selected from the Journal of Microbes and Infections (China)}

Copyright (C) 2010 by World Scientific Publishing Co. Pte. Ltd.

All rights reserved. This book, or parts thereof, may not be reproduced in any form or by any means, electronic or mechanical, including photocopying, recording or any information storage and retrieval system now known or to be invented, without written permission from the Publisher.

For photocopying of material in this volume, please pay a copying fee through the Copyright Clearance Center, Inc., 222 Rosewood Drive, Danvers, MA 01923, USA. In this case permission to photocopy is not required from the publisher.

ISBN-13 978-981-283-566-6

ISBN-10 981-283-566-0

Typeset by Stallion Press

Email: enquiries@stallionpress.com

Printed in Singapore. 


\section{Preface I}

Infectious diseases are of global concern. They have been such in the past and will continue to be such in the future. Multidrug-resistant tuberculosis, malaria, AIDS, viral hepatitis, pandemic influenza and other emerging infections are just a few of the problems the world is facing. For China, the country with the largest population in the world, most of these diseases are a particular challenge. A dramatic example is the SARS outbreak, which has told us that, in order to successfully control infections, increased efforts are necessary to understand the disease mechanisms and epidemiology and to develop new tools for diagnosis, prophylaxis and therapy. This volume is an impressive document on the enormous progress that Chinese research has made in the last couple of years in all of these fields. Since, so far, only some of these studies have been published in international journals, a lot of information has not spread beyond the borders of the country. I therefore warmly welcome the opportunity to introduce this volume, which gives a broader overview to the outside world of what Chinese bacteriologists and virologists are doing.

Hans Dieter Klenk Universität Marburg, Germany October 28, 2008 
This page intentionally left blank 


\section{Preface 2}

Thirty years ago, when China resumed its normal environment for scientific activities, communication with the outside world began. A number of eminent virologists, bacteriologists and molecular biologists, including the late Joseph Melnick, Pierre Tiollais, Morris Pollard and my many other friends, visited China, and I had the opportunity to be their scientific interpreter. Besides, young Chinese students and scientists were sent abroad to study, which was hugely beneficial for China in proceeding at a rapid pace during the 30 years. In 1982, my mentor, the late Prof. Fei-Qin Lin, started to edit a journal named Medical Microbiology Abroad, which published reviews and abstracts introducing new progress in medical microbiology from journals published in various languages, including English, French, Russian and Japanese. The editorial board of Medical Microbiology Abroad consisted of medical microbiologists from many provinces of China, and the journal was well received. Medical Microbiology Abroad has been an important reference journal providing scientific information on medical microbiology for scientists and mostly graduate students throughout China, especially for those in regions where foreign journals were not easily accessible.

After more than 20 years of publishing Medical Microbiology Abroad, the editorial board realized that since the Internet is widely used, people can read journals on the websites. At the same time, more and more research on medical microbiology is being conducted, and to promote translational research we need to set up close contact among microbiologists, clinicians, veterinarians, epidemiologists, vaccinologists and health workers. Therefore, we decided to change Medical Microbiology Abroad into the Journal of Microbes and Infections, a journal publishing research articles, reviews and scientific news on microbes and infections in humans and animals. The current editorial board has been expanded to include microbiologists, immunologists, epidemiologists, medical doctors 
and veterinarians. The first issue of the Journal of Microbes and Infections (in Chinese) was published in 2006. After close to three years of hard work, we have been looking for opportunities to introduce part of the work in China to the outside world. Now, our effort is supported by World Scientific Publishing Company, which decided to publish selected articles from the journal in English. We consider this a good approach to building up communication between Chinese scientists and scientists abroad. We hope that this window will open up a supplementary track for scientists outside of China to know in detail the work on microbes and infections carried out in China, and perhaps this journal can also serve as a matchmaker for future collaboration among scientists. Due to the limitation of space, the material and methods are omitted from this book. However, readers can contact the authors for details.

Yu-Mei Wen Key Laboratory of Medical Molecular Virology Institute of Medical Microbiology Institutes of Biomedical Sciences

Shanghai Medical College Fudan University, Shanghai, China 


\section{Contents}

Preface 1

Hans Dieter Klenk

Preface 2

Yu-Mei Wen

Section 1. Bacteria

Introduction and Comments: Studies on Bacteria by Molecular and Experimental Approaches

Yu-Mei Wen

1.1. Cloning, Expression and Immunological Evaluation of a Novel Protein Belonging to the OmpA Family in Leptospira interrogans Wei Lin, Hong-Liang Yang, Yang Yang, Bao-Yu Hu, Li-Zhi Tan and Xiao-Kui Guo

1.2. Surveillance of Leptospirosis and the Murine Reservoirs in China, 2005

Xiu-Gao Jiang, Xiang-Yan Zhang, Wen-Jun Zhong, Yi-Xin Nie, Wen-Wu Yin, Xiu-Wen Li and Xiao-Kui Guo

1.3. Cloning and Expression of the Histidinol Dehydrogenase Gene from Mycobacterium tuberculosis H37Rv and Properties of the Recombinant Histidinol Dehydrogenase

Rui-Liang Jin, Rui-Qing Liu, Hong-Jun Wang, Jian Cao, Sheng-Feng Xu, Li-Juan Chunyu, Yun-Min Xu and Hong-Hai Wang 
1.4. Cloning and Sequence Analysis of the fbps Gene

of Streptococcus suis Type 2

Li-Yun Sun, Hong-Jie Fan and Cheng-Ping Lu

1.5. Effects of EnvZ Histidine Kinase Inhibitors on the Virulence of Shigella flexneri Ming-Liang Chen, Qi Wang, Sheng-Fu Dong, Zhi-Qiang Qin, Jian Zhang, Cong Han, Xia Cai, $X u$ Shen and Di Qu

1.6. Detection of Virulence and Genes for Viable but Nonculturable (VBNC) Campylobacter jejuni Jie-Lin Yang, Tong-Hai Dou, Ming Gu and Zhong-Liang Wu

Section 2. Viruses

Introduction and Comments: Trends on Virus Research in China

Yu-Mei Wen

2.1. Transcriptomic and Proteomic Studies on HBsAg-Positive Transgenic Mouse Livers Suggest Alterations in Carbohydrate, Lipid and Amino Acid Metabolism Jun Ren, Chao Zhao, Cai-Yun Fang, Zhang-Mei Ma, Peng-Yuan Yang and Yu-Mei Wen

2.2. Large-Scale Polymorphism Analysis of Hepatitis B Virus Envelope Protein using Bioinformatics Zhe Chen, Yue Zhu, Yi-Xue Li and Yu-Mei Wen

2.3. Studies on the Inactivation of Hepadnavirus in Blood Components by Using a Duck Hepatitis B Virus-Infected Animal Model Wen-Yi Wang, Yu-Wen Huang, Ping Lu, Jian-Er Long, Qin Mo, Bing Ling, Xiao-Ling Han, Sheng-Fu Dong, Di Qu and Kai-Cheng Qian 
2.4. Sequence Analysis of the SH Gene and Its Flanking Region of a Mumps Virus Strain (SP Strain)

Shao-Hui Ma, Rong-Song Zhang, Long-Ding Liu, Jing-Jing Wang, Li-Chun Wang, Wen-Jun Yang, Yan Liang, Zi-Jiang Yang and Qi-Han Li

2.5. HIV-Related Knowledge, Attitudes and Behaviors Among Policewomen and Female Prisoners from a Behavior Correction Center Wei Jiao, Ying-Jie Zheng, Xue-Cai Wang, Ai-Qin Xu, Jian-Ping Gu, Jian-Fu Zhu, Yi-Han Lu, Fa-Di Wang and Qing-Wu Jiang

\section{Section 3. Laboratory Medicine}

Introduction and Comments: Advances and Trends in Diagnostic Microbiology in China Yi-Wei Tang

3.1. Detection of SARS-Associated Coronavirus Infection by Reverse Transcriptase-Polymerase Chain Reaction, Indirect Immunofluorescence Assay and Enzyme-Linked Immunosorbent Assay Zhi-Tong Zhou, Wei-Lun Jiang, Zheng-Hong Yuan, Fang Shen, Di Qu, Shi-Min Gu, Long-Ting Fu and Ren-Fang Zhang

3.2. Bacteriological and Molecular Identification of a Sporadic Case of Brucellosis in Shanghai Cheng-Yan Meng, Fei-Yi Ruan, Jian-Qiang Lei, Jia-Lin Jin and Wen-Hong Zhang

3.3. A PCR-Capillary Electrophoresis Method Detects and Differentiates Biovars of Ureaplasma urealyticum Shi-Hang Zhou and Zhuo-Ran Zhang

3.4. Implications of CD64 in Early Diagnosis of Neonatal Septic Cases Rong Zhang and Chao Chen 
3.5. Correlation Between the MICA*A5.1 Allele

and Chlamydia trachomatis Infection

Bing Mei, Kun Du, Zhi Huo, Fu-Yan Wang,

Shu-Hui Ma and Ping Yu

3.6. Surveillance of Human Infections with Avian Influenza in Guangzhou During 1997-2006

Peng-Zhe Qin, Yu-Lin Wang, Biao Di, Xiu-Zhen Zhou, Yang Liu, Yan-Shan Cai, Ji-Chuan Shen, Yu-Fei Liu, En-Jie Lu, Duan-Hua Zhou and Ming Wang

3.7. Clinical Significance of Human Cytomegalovirus pp65-Specific Antibodies for Verification of HCMV Primary Infection Qian Liu, Jing-Xian Chen and Ming-Li Wang

3.8. Analysis of Indeterminate HIV Western Blot Profiles Kai Gao, Yan Li, Cai-Yun Liang, Hui-Fang Xu and Zhi-Gang Han

3.9. Detection of Multidrug-Resistant Mycobacterium tuberculosis by Sputum Smears Shu-Yan Wu, Guo-Hao Gu and Rui Huang

Section 4. Clinical Medicine

Introduction and Comments: A Glimpse of Clinical

Infectious Diseases in China in the

\section{New Millennium}

Shan Lu

4.1. The Role of Lower Airway Bacterial Colonization at the Stable Stage of Chronic Obstructive Pulmonary Disease Min Zhang, Xin Zhou, Xin-Yi Zhang and Xing Ding

4.2. Gene Sequence Analysis of the Norovirus Detected in Diarrhea Specimens from Children in a Surveillance Hospital in Shanghai Zheng Teng, Xi Zhang, Kang-Sheng Wen, Jun-Jie Shao and Bai-Hui Zhao 
4.3. Clinical Analysis of 77 Measles Patients Dong-Mei Shi and Qing Xie

4.4. Analysis of Clinical Characteristics in

77 HIV-Infected Individuals

Qi-Ming Gong, De-Qi Qiu, Zhi-Meng Lu

and Xin-Xin Zhang

4.5. Etiology and Clinical Characteristics of Children

Hospitalized for Acute Lower Respiratory Tract

Infection in Winter

Jun Shen, Qi-Rong Zhu, Xiao-Hong Wang

and Chuan-Qin Wang

4.6. Infectious Disease Risks Associated with the

Sichuan Earthquake: Experiences in Treatment

of Earthquake Wound Infections

Xin Zhao, Wen-Sheng Xu, Jun-Xue Wang,

Xiao-Hui Miao and Yu-Dong Gu

\section{Section 5. Immunology and Vaccinology}

Introduction and Comments: Exploring Host Immune

\section{Responses to Microbes}

Shan Lu

5.1. Detection of Dendritic Cell Subsets in Peripheral

Blood and Levels of IL-12 and IFN- $\gamma$ in Serum of Patients with Syphilis Li-Xiong Zheng, Si-Ning Fang, Fang-Juan Li, Xiao-Hong Du, Qiu-Sheng Tong, Ya-Jie Zheng, Hui Qi and Xin-Gen Wang

5.2. Analysis of Antigenicity and Immunogenicity

of the Receptor-Binding Domain of SARS-CoV

Ping Zhao, Jin-Shan Ke, Min Liu, Wei Pan and Zhong-Tian Qi 
5.3. Alterations in Chemokine Expression in PBMCs

During and After Interferon- $\alpha$ Therapy in HCV

Chronic Infections

Yun-Wen Hu, Jian Wang, Ji-Ming Zhang, Yi Xie and Zheng-Hong Yuan

5.4. A Summary of Immunogenicity Studies on DNA

Vaccines Expressing the Hepatitis B Virus Core and Surface Antigens as Tested in Both Murine and Nonhuman Primate Models

Yi-Ping Xing, Zu-Hu Huang, Shi-Xia Wang, Jun Li and Shan Lu

List of Contributors

Index 